\title{
Robotic versus conventional laparoscopic surgery for rectal cancer: systematic review and meta-analysis
}

\author{
Seon Heui Lee, Sungwon Lim', Jin Hee Kim², Kil Yeon Lee ${ }^{3}$ \\ Department of Nursing Science, College of Nursing, Gachon University, Incheon, ${ }^{1}$ National Evidence-based Healthcare \\ Collaborating Agency, Seoul, '2Department of Nursing, College of Medicine, Chosun University, Gwangju, ${ }^{3}$ Department of Surgery, \\ Kyung Hee University School of Medicine, Seoul, Korea
}

\begin{abstract}
Purpose: Robotic surgery (RS) overcomes the limitations of previous conventional laparoscopic surgery (CLS). Although meta-analyses have been published recently, our study evaluated the latest comparative surgical, urologic, and sexual results for rectal cancer and compares RS with CLS in patients with rectal cancer only.

Methods: We searched three foreign databases (Ovid-MEDLINE, Ovid-Embase, and Cochrane Library) and five Korean databases (KoreaMed, KMbase, KISS, RISS, and KisTi) during July 2013. The Cochrane Risk of Bias and the Methodological Index for Non-Randomized were utilized to evaluate quality of study. Dichotomous variables were pooled using the risk ratio (RR), and continuous variables were pooled using the mean difference (MD). All meta-analyses were conducted with Review Manager, V. 5.3.

Results: Seventeen studies involving 2,224 patients were included. RS was associated with a lower rate of intraoperative conversion than that of CLS (RR, 0.28; 95\% confidence interval [CI], 0.15-0.54). Time to first flatus was short (MD, -0.13 ; $95 \% \mathrm{Cl},-0.25$ to -0.01 ). Operating time was longer for RS than that for CLS (MD, 49.97; 95\% Cl, 20.43-79.52, $\mathrm{I}^{2}=97 \%$ ). International Prostate Symptom Score scores at 3 months better RS than CLS (MD, $-2.90 ; 95 \% \mathrm{Cl},-5.31$ to $-0.48, \mathrm{I}^{2}=0 \%$ ). International Index of Erectile Function scores showed better improvement at 3 months (MD, $-2.82 ; 95 \% \mathrm{Cl},-4.78$ to -0.87 , $\mathrm{I}^{2}=37 \%$ ) and 6 months (MD, $-2.15 ; 95 \% \mathrm{Cl},-4.08$ to $-0.22, \mathrm{I}^{2}=0 \%$ ).

Conclusion: RS appears to be an effective alternative to CLS with a lower conversion rate to open surgery, a shorter time to first flatus and better recovery in voiding and sexual function. RS could enhance postoperative recovery in patients with rectal cancer.

[Ann Surg Treat Res 2015;89(4):190-201]
\end{abstract}

Key Words: Robotic surgical procedures, Rectal neoplasms

\section{INTRODUCTION}

Laparoscopic colorectal surgery has been proven to be as safe and effective as open surgery and offers various advantages [1]. Conventional laparoscopic surgery (CLS) provides good magnification and illumination for colorectal surgeons, which improve visualization of pelvic structures [2]. CLS for patients with colorectal diseases results in better short-term morbidity than that from open surgery [3]. Although CLS has revolutionized surgical management of colorectal cancers over the past two decades, it has several technical limitations, particularly when excising rectal cancer, including a limited range of instrument motion in the narrow pelvic cavity, related loss of dexterity, and an inadequate visual field associated with an unstable camera view [1,4]. Robotic systems have been widely adopted for urological, gynecological, and rectal surgery. In particular, a robotic system is favorable for deep and narrow spaces, such as the pelvis or mediastinum [5,6].
Received March 5, 2015, Revised June 13, 2015, Accepted July 4, 2015

\section{Corresponding Author: Kil Yeon Lee}

Department of Surgery, Kyung Hee University Medical Center, Kyung Hee University School of Medicine, 23 Kyungheedae-ro, Dongdaemun-gu, Seoul 02447, Korea

Tel: +82-2-958-8241, Fax: +82-2-966-9366

E-mail: isaac34@korea.com
Copyright (c) 2015, the Korean Surgical Society

(c) Annals of Surgical Treatment and Research is an Open Access Journal. All articles are distributed under the terms of the Creative Commons Attribution NonCommercial License (http://creativecommons.org/licenses/by-nc/4.0/) which permits unrestricted non-commercial use, distribution, and reproduction in any medium, provided the original work is properly cited. 
Since the first robot-assisted colectomy was performed in 2001 [7], a number of studies have been published on the use of robotic systems in colorectal surgery. In particular, robotic surgery (RS) overcomes the limitations of previous CLS, including a motion filter for tremor-free surgery, high definition three-dimensional imaging, an easily controlled camera on a stable platform, and increased space for the operating instruments [8]. Currently, Baik et al. [9] reported the feasibility and safety of robot-assisted tumor-specific mesorectal excision of rectal cancer in terms of oncologic outcomes. Also, Bae et al. [10] reported robotic pelvic lymph node dissection for rectal cancer was feasible and safe. A few systematic reviews and meta-analyses comparing outcomes between RS and CLS have been conducted [11-14]. However, there is some limitations of the studies because they analyzed safety and efficacy but included noncomparative studies or mixed colonic and rectal cancers.

Therefore, we evaluated the safety and efficacy of RS by performing a systematic review and meta-analysis focusing on rectal cancer studies that compared RS and CLS.

\section{METHODS}

\section{Search strategy and study selection}

We searched three foreign databases (Ovid-MEDLINE, OvidEmbase, and Cochrane Library) and five Korean databases (KoreaMed, KMbase, KISS, RISS, and KisTi) on July 23, 2013. We searched pertinent MeSH or EMTREE terms (rectal neoplasms, rectal cancer, and rectal carcinoma or adenocarcinoma or tumor), intervention terms (robotics, computer-assisted surgery, telerobot, remote operation, remote surgery, and Da Vinci) and their combinations. No language was restricted, and no filters were applied. The references from published reviews were also assessed to seek additional publications. A total of 1,664 records were ultimately identified through the search. After removing duplicates, 1,302 studies were imported for the first title and abstract screening.

A total of 54 articles were obtained to conduct the full-text review, and 23 articles were identified to be potentially relevant. We excluded 9 of 23 publications because 4 may have had overlapping cohorts, and 5 studies did not report appropriate results. We considered sample size, publication year, study design, and the number of outcomes of interest to choose highquality data with a low risk of bias for the publications with possible overlap. Finally, we add 3 articles by hand searching. Total seventeen publications were ultimately selected for the meta-analysis (Fig. 1).

\section{Inclusion and exclusion criteria}

Two authors (S.L. and J.H.K.) independently reviewed all abstracts. We retrieved full-text copies of all studies that potentially met the inclusion criteria based on a review of the abstract. If both authors agreed that a study did not meet the eligibility criteria, we excluded the study. If we disagreed, we resolved the conflict by discussion and consensus or by consulting a third review team member. The inclusion criteria were: (1) compared outcomes of RS and CLS in patients with rectal cancer and (2) if multiple studies were reported for the same patient population, either the one of higher quality or the most recent study was used. Studies fulfilling the following criteria were excluded: (1) the outcomes and parameters of patients with rectal cancer were not reported clearly; (2) it was impossible to extract appropriate data from the published results; (3) the studies were not original articles and included gray literature, such as letters, editorials, and expert opinions or reviews without original data; and (4) abstracts, theses, casereports, and studies lacking a control group.

\section{Data extraction and quality assessment}

Each author extracted the data independently from each study and compared them, resolving disagreement by discussion: (1) first author and year of publication; (2) study design; (3) country in which the study was performed; (4) number of subjects operated on with each technique; (5) study population demographic and clinical characteristics; (6) clinical outcomes, conversion rates, time to oral diet, time to bowel function recovery, estimated blood loss (EBL), bowel obstruction, complications (anastomotic leakage, urinary retention, intrapelvic abscess, or intrapelvic bleeding); (7) oncologic outcomes (number of lymph nodes extracted); and (8) urological and sexual function (e.g., International Prostate Symptom Score [IPSS] and International Index of Erectile Function [IIEF]).

Two authors (S.L. and J.H.K.) assessed all of the studies included for methodological quality according to the Risk of Bias for Randomized Controlled Trials (RCTs) [15] and the Methodological Index for Non-randomized Studies (MINORS) for nonrandomized comparative studies [16]. The information is summarized in Table 1 and Fig. 2.

\section{Statistical analysis}

Dichotomous variables were pooled using the risk ratio $(\mathrm{RR})$, and continuous variables were pooled using the mean difference (MD). RR and MD were calculated and reported with 95\% confidence intervals (CIs). Statistical heterogeneity among the studies was analyzed using the chi-square and Q-tests [17]. A fixed effects model was used for studies with low or moderate statistical heterogeneity $\left(\mathrm{I}^{2}<25,25 \%-50 \%\right)$, and a random effects model was used for studies with high statistical heterogeneity $\left(\mathrm{I}^{2}>50 \%\right)$. Meta-analyses of dichotomous variables were performed using the Mantel-Haenszel (M-H) method, and continuous variables were analysed using the 


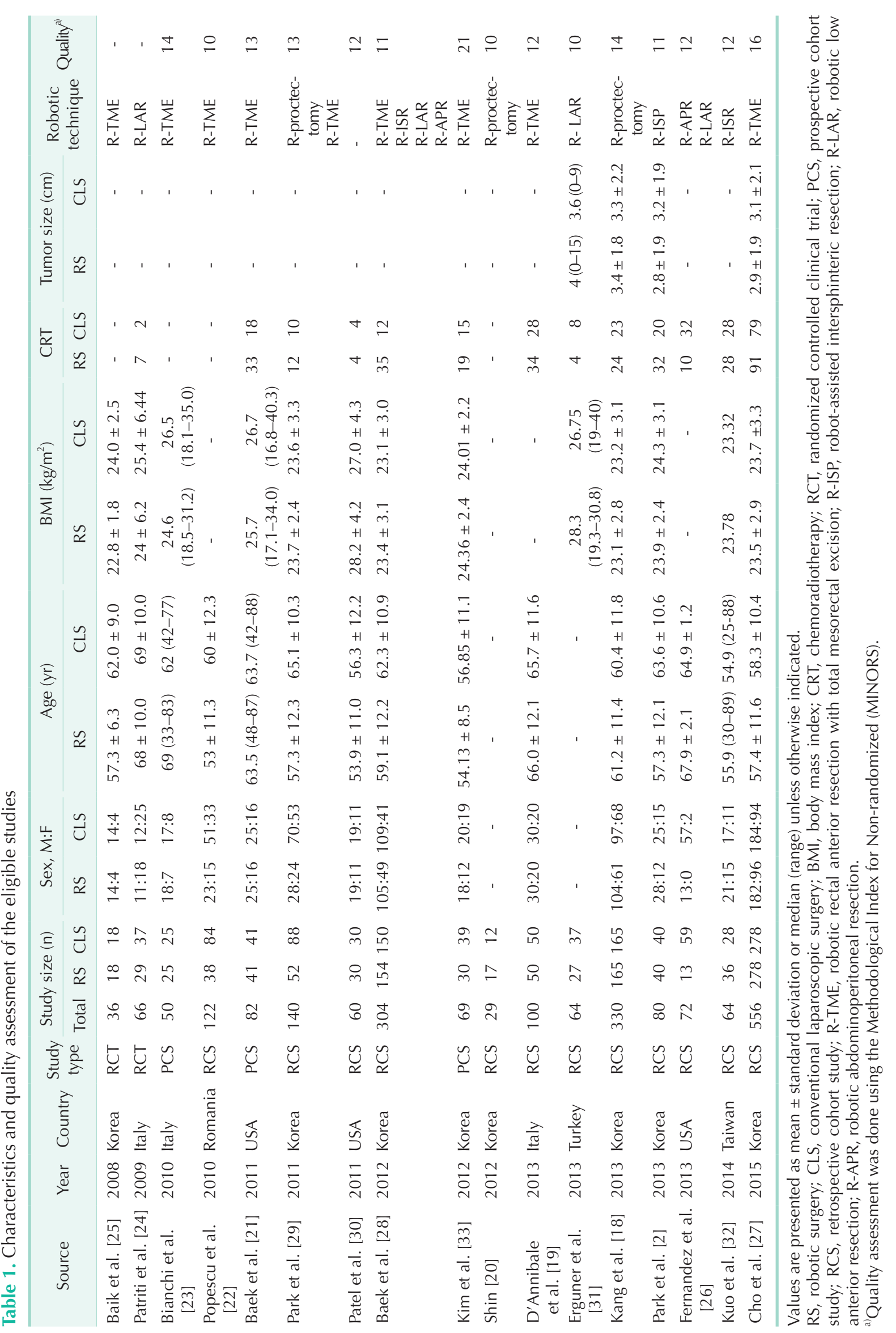




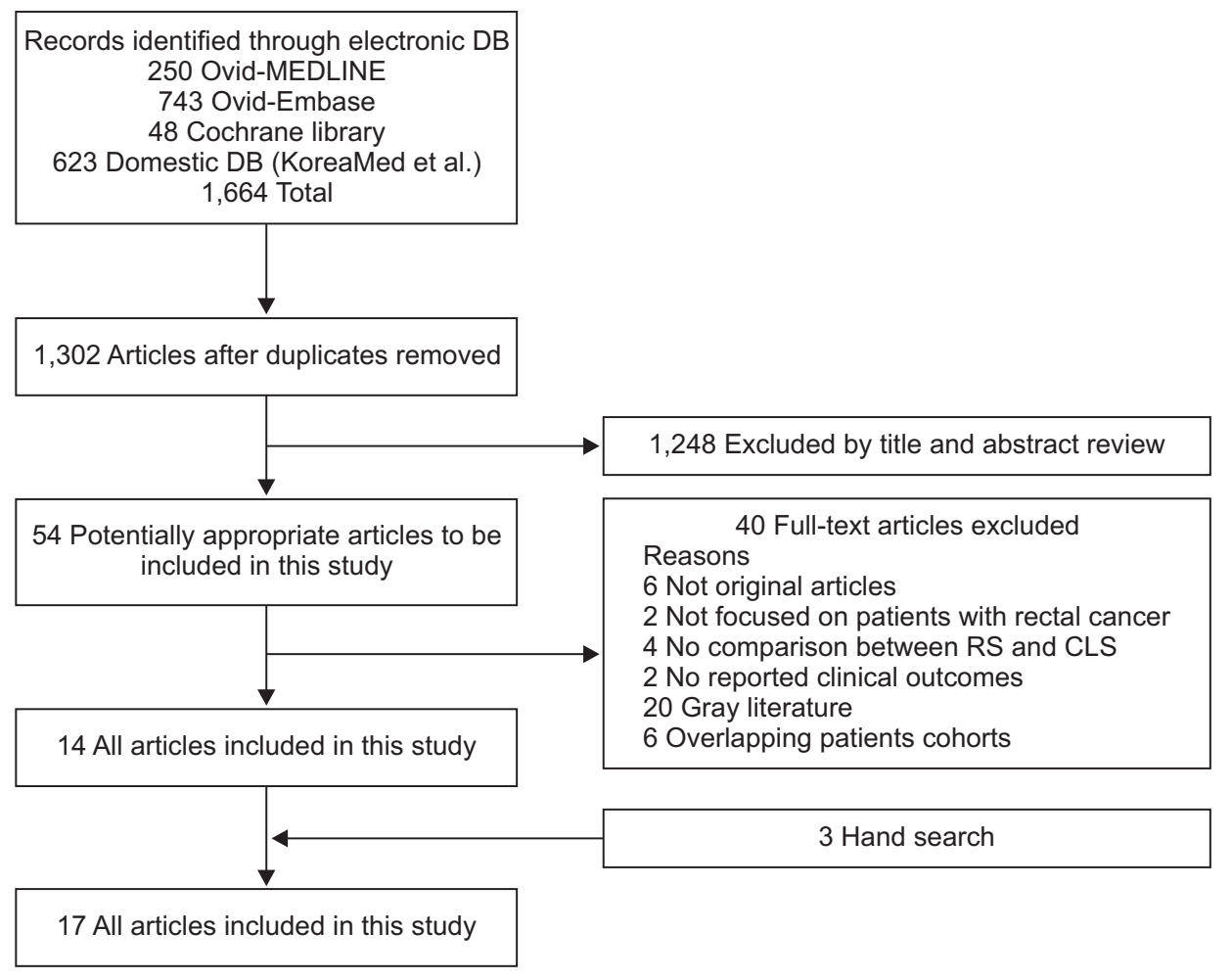

Fig. 1. Flow diagram of the study selection process. RS, robotic surgery; CLS, conventional laparoscopic surgery. inverse variance method. We conducted all meta-analyses with Review Manager, V. 5.3 (RevMan, Copenhagen, Denmark: The Nordic Cochrane Centre, The Cochrane Collaboration, 2014) and used the two-tailed test of significance $(\mathrm{P}<0.05)$.

\section{Ethical consideration}

This study was reviewed and approved by the Institutional Review Board at the National Evidence-Based Healthcare Collaborating Agency (NECA): (approval number: NECA IRB 13013)

\section{RESULTS}

\section{Study characteristics}

Table 1 shows the characteristics of the 17 studies and their patients. The 17 studies included 2,224 patients: 1,043 in the RS group and 1,181 in the CLS group. Two studies were RCTs, four were prospective cohort studies, and eleven were retrospective cohort studies. Of the 17 studies, 8 were conducted in Korea, 3 were conducted in Italy, 3 in the USA, 1 in Turkey, 1 in Taiwan, and 1 in Romania. All studies were published during the last 7 years (2008-2015). The risk of bias in the RCT studies was high (Fig. 2). The quality of the cohort studies, except Kim (2012), was poor (Table 1).

\section{Conversion rates}

Ten studies [18-27] reported a conversion rate to open

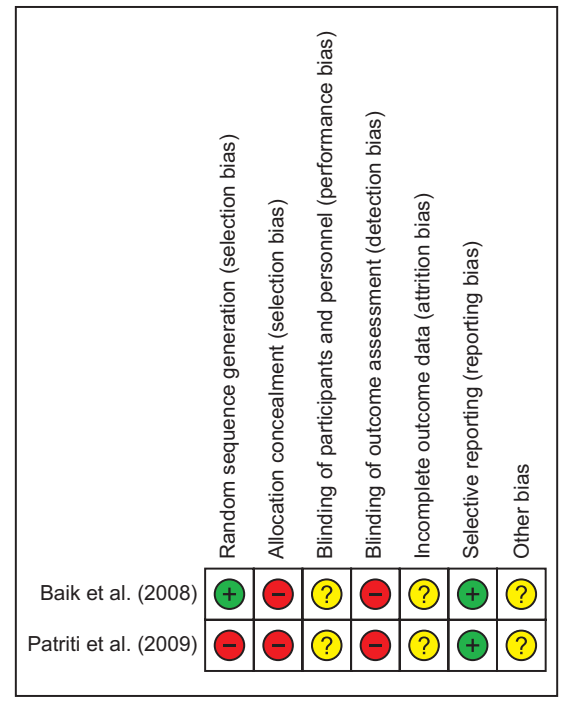

Fig. 2. The risk of bias for eligible randomized controlled trials.

surgery. In all, 8 patients (1.19\%) undergoing RS and 50 (6.50\%) undergoing CLS required conversion to open surgery (RR, 0.28; $95 \% \mathrm{CI}, 0.15-0.54$ ), with no heterogeneity among studies ( $\mathrm{P}=$ $0.98, I^{2}=0 \%$ ) (Table 2, Fig. 3).

\section{Time to oral diet}

Time to liquid diet was reported in six studies [2,18,22,2729] comparing RS and CLS. The pooled estimates using a fixed 


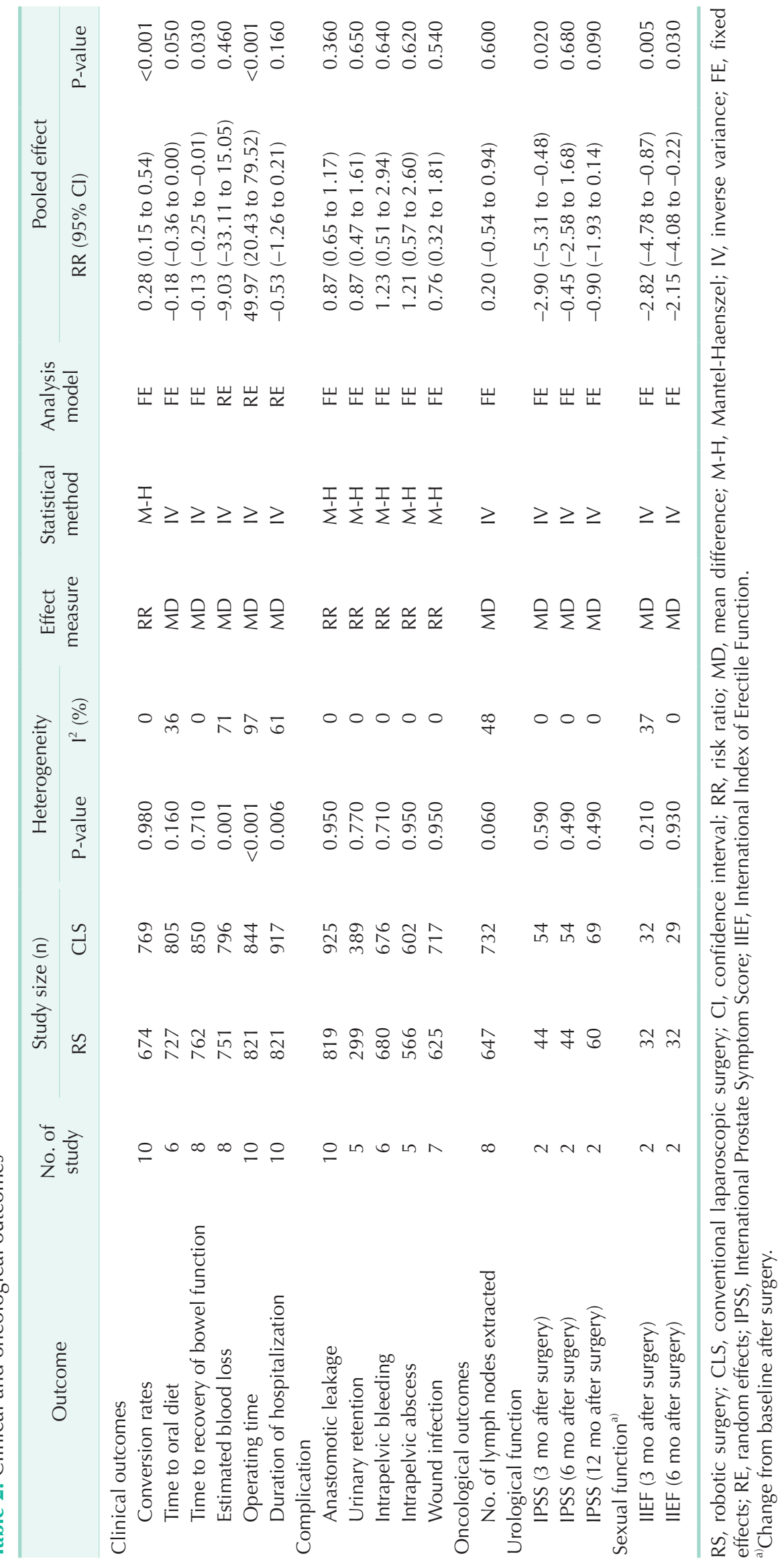




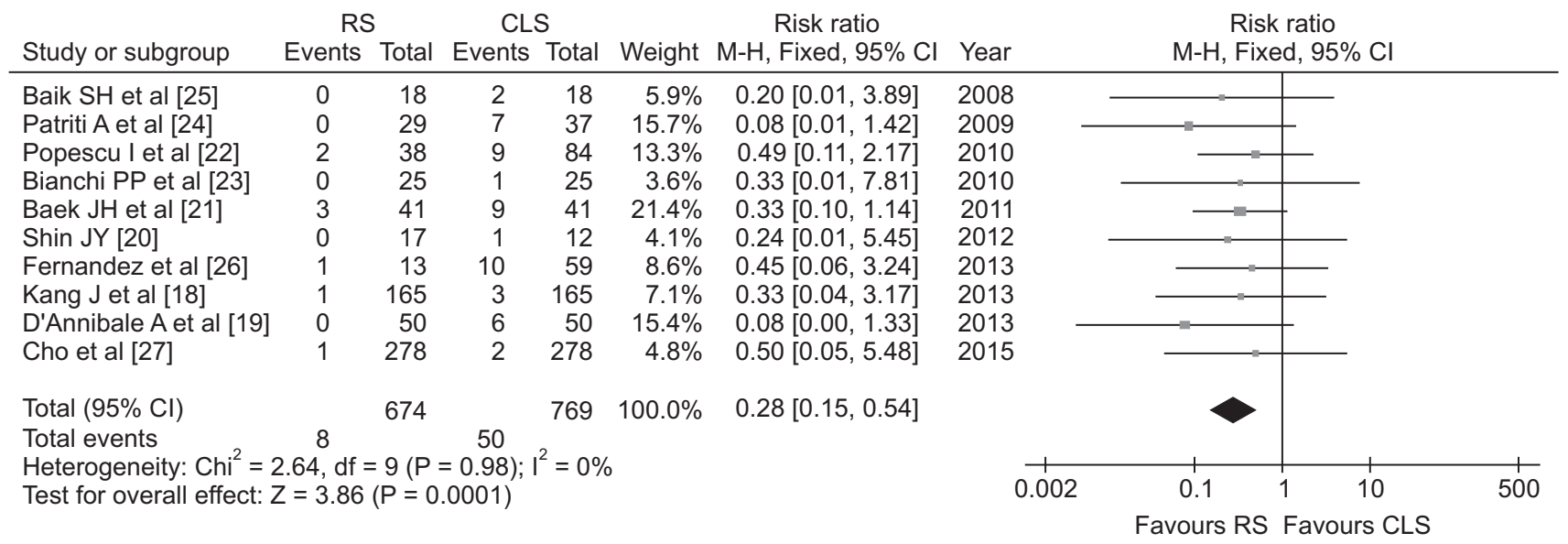

Fig. 3. Forest plot and meta-analysis of conversion to open surgery. RS, robotic surgery; CLS, conventional laparoscopic surgery; M-H, Mantel-Haenszel; Cl, confidence interval; df, degree of freedom.

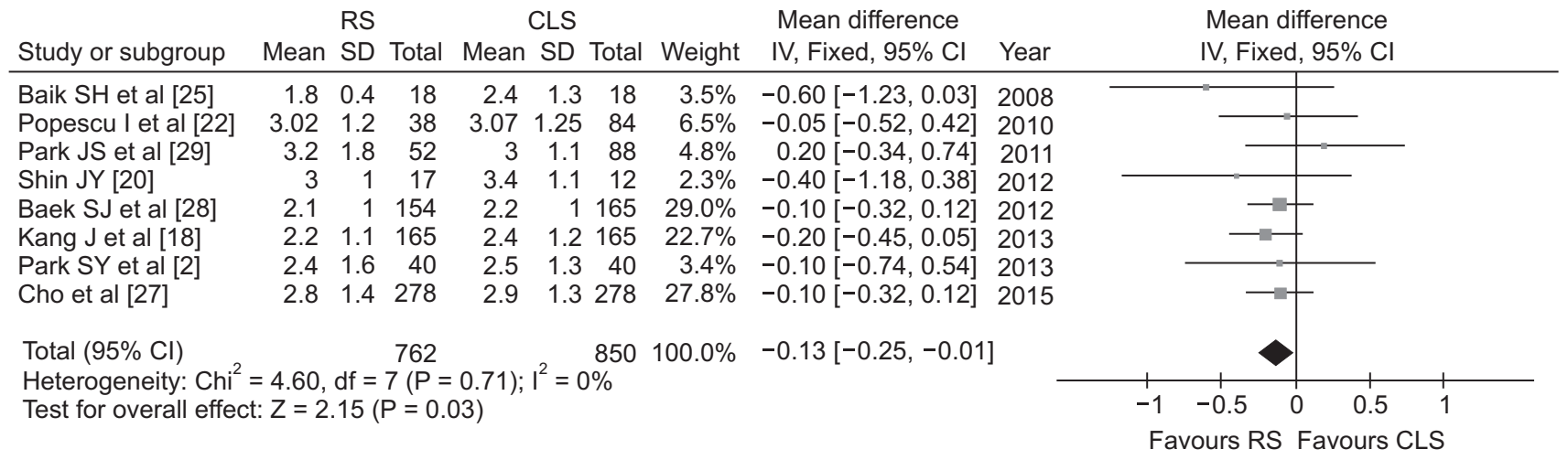

Fig. 4. Forest plot and meta-analysis of time to first flatus. RS, robotic surgery; CLS, conventional laparoscopic surgery; SD, standard deviation; $\mathrm{Cl}$, confidence interval; df, degree of freedom.

effects model showed no significant difference between RS and CLS (MD, -0.18 days; $95 \%$ CI, -0.36 to 0.00 ), with moderate heterogeneity $\left(\mathrm{P}=0.16, \mathrm{I}^{2}=36 \%\right.$ ) (Table 2$)$.

\section{Time to bowel function recovery}

Eight studies [2,18,20,22,25,27-29] analyzed time to first flatus. The pooled estimates using a fixed effects model showed a significant difference between RS and CLS (MD, -0.13 days, $95 \% \mathrm{CI},-0.25$ to -0.01 ), with no heterogeneity among studies ( $\mathrm{P}$ $=0.71, I^{2}=0 \%$ ) (Table 2, Fig. 4).

\section{Estimated blood loss}

EBL was reported in eight studies $[2,18,20,22,24,27,28,30]$ comparing RS and CLS. The pooled estimates using a random effects model showed no significant difference between RS and CLS (MD, $-9.03 \mathrm{~mL}$; 95\% CI, -33.11 to 15.05), with heterogeneity ( $\mathrm{P}=0.001, \mathrm{I}^{2}=71 \%$ ) (Table 2$)$.

\section{Operating time}

Operating time was reported in ten studies $[2,18,20,22,24,25$,
27-30] comparing RS and CLS. The pooled estimates using a random effects model showed significantly longer operating time for RS than that for CLS (MD, 49.97 minutes; 95\% CI, 20.43-79.52). However, this result had high heterogeneity $(\mathrm{P}<$ $0.001, I^{2}=97 \%$ ) (Table 2, Fig. 5).

\section{Duration of hospitalization}

Hospitalization duration was reported in ten studies $[2,18,20,22,24,25,27-30]$ comparing RS and CLS. The pooled estimates using a random effects model showed no significant difference between RS and CLS (MD, -0.53 days; $95 \% \mathrm{CI},-1.26$ to 0.21$)$, with heterogeneity $\left(P=0.006, I^{2}=61 \%\right)$ (Table 2).

\section{Complications}

Anastomotic leakage

Anastomotic leakage was reported in ten studies [2,18,19,2224,26-28,31] comparing RS and CLS. No significant difference in bowel anastomotic leaks was observed between RS and CLS (RR, 0.87; 95\% CI, 0.65-1.17), with no heterogeneity ( $\left.\mathrm{P}=0.95, \mathrm{I}^{2}=0 \%\right)$ (Table 2). 


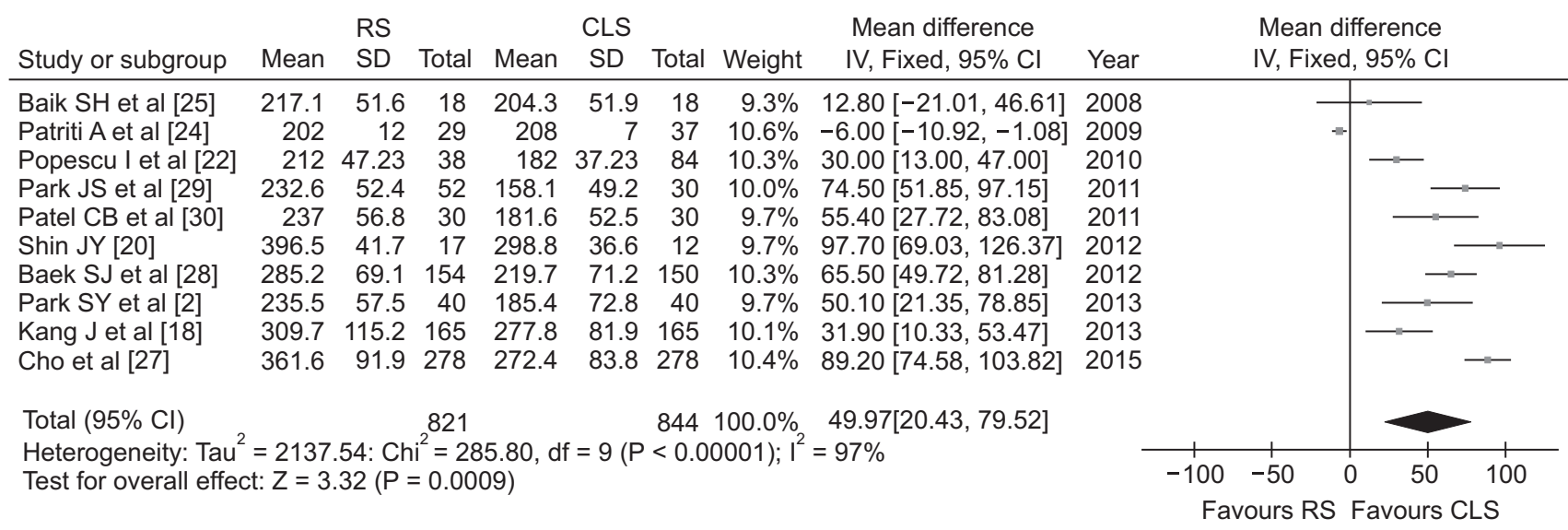

Fig. 5. Forest plot and meta-analysis of operation time. RS, robotic surgery; CLS, conventional laparoscopic surgery; SD, standard deviation; $\mathrm{Cl}$, confidence interval; $\mathrm{df}$, degree of freedom.

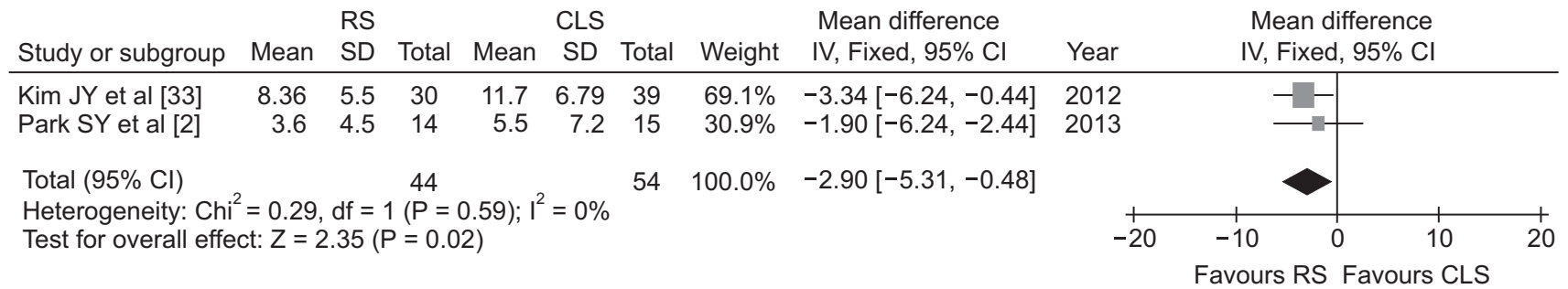

Fig. 6. Forest plot and meta-analysis of International Prostate Symptom Score 3 months after surgery. RS, robotic surgery; CLS, conventional laparoscopic surgery; SD, standard deviation; $\mathrm{Cl}$, confidence interval; df, degree of freedom.

\section{Urinary retention}

Urinary retention was reported in five studies $[2,18,24,26,29]$ comparing RS and CLS. The pooled estimates using a fixed effects model showed no significant difference between RS and CLS (RR, 0.87; 95\% CI, 0.47-1.61), with no heterogeneity ( $\mathrm{P}=0.77$, $\mathrm{I}^{2}=0 \%$ (Table 2).

\section{Intrapelvic bleeding}

Intrapelvic bleeding was reported in six studies $[18,24,25$, 27,28,32] comparing RS and CLS. The pooled estimates using a fixed effects model showed no significant difference between RS and CLS (RR, 1.23; 95\% CI, 0.51-2.94), with no heterogeneity (P $=0.71, \mathrm{I}^{2}=0 \%$ (Table 2).

\section{Intrapelvic abscess}

Intrapelvic abscess was reported in five studies $[18,21,27$, 29,30] comparing RS and CLS. The pooled estimates using a fixed effects model showed no significant difference between $\mathrm{RS}$ and CLS (RR, 1.21; 95\% CI, 0.57-2.60), with no heterogeneity ( $\mathrm{P}$ $\left.=0.95, \mathrm{I}^{2}=0 \%\right)($ Table 2$)$

\section{Wound infection}

Wound infection was reported in seven studies $[2,18,22,23,27$, 29,31] comparing RS and CLS. The pooled estimates using a fixed effects model showed no significant difference between RS and CLS (RR, 0.76; 95\% CI, 0.32-1.81), with no heterogeneity $\left(\mathrm{P}=0.95, \mathrm{I}^{2}=0 \%\right)($ Table 2$)$.

\section{Number of lymph nodes extracted}

Eight studies [18-20,22,24,25,27,29] reported the numbers of lymph nodes extracted. No significant differences were observed in the total numbers of lymph nodes extracted between patients undergoing RS and CLS (MD, 0.20; $95 \% \mathrm{CI},-0.54$ to 0.94$)$, with no heterogeneity $\left(\mathrm{P}=0.06, \mathrm{I}^{2}=48 \%\right.$ ) (Table 2).

\section{Urological function (IPSS)}

Urological function was reported in three studies $[2,19,33]$ comparing RS and CLS. The outcome was evaluated using IPSS with high scores corresponding with greater dysfunction. At three months follow-up, the pooled estimates using a fixed effects model showed significant difference of IPSS scores between RS and CLS (MD, $-2.90 ; 95 \% \mathrm{CI},-5.31$ to -0.48 ), with no heterogeneity $\left(\mathrm{P}=0.59, \mathrm{I}^{2}=0 \%\right.$ ) (Table 2, Fig. 6). At six month three months follow-up, the pooled estimates showed a no significant difference of IPSS scores between RS and CLS ( $\mathrm{MD},-0.45 ; 95 \% \mathrm{CI},-2.58$ to 1.68$)$, with no heterogeneity ( $\mathrm{P}=$ $0.49, \mathrm{I}^{2}=0 \%$ ) (Table 2). Lastly, at the 12 months, there was no significant difference of IPSS score between RS and CLS (MD, 


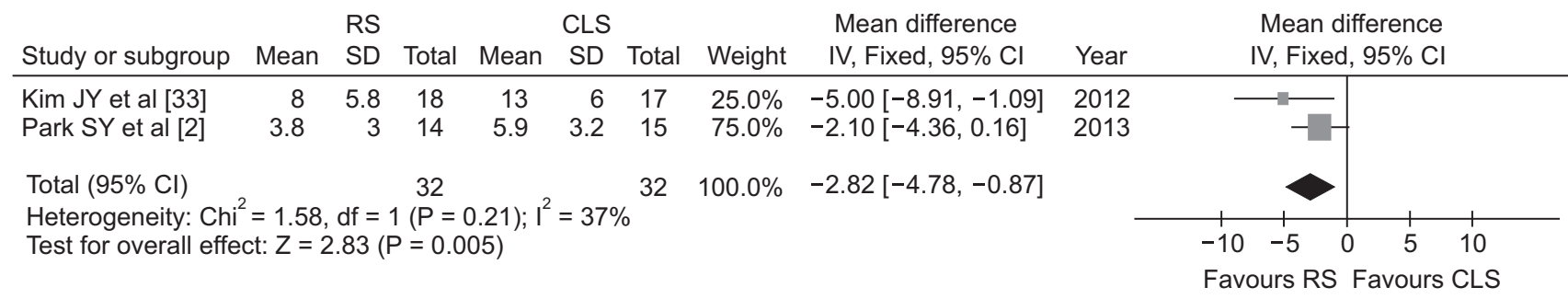

Fig. 7. Forest plot and meta-analysis of International Index of Erectile Function score change from baseline to 3 months after surgery. RS, robotic surgery; CLS, conventional laparoscopic surgery; SD, standard deviation; $\mathrm{Cl}$, confidence interval; df, degree of freedom.

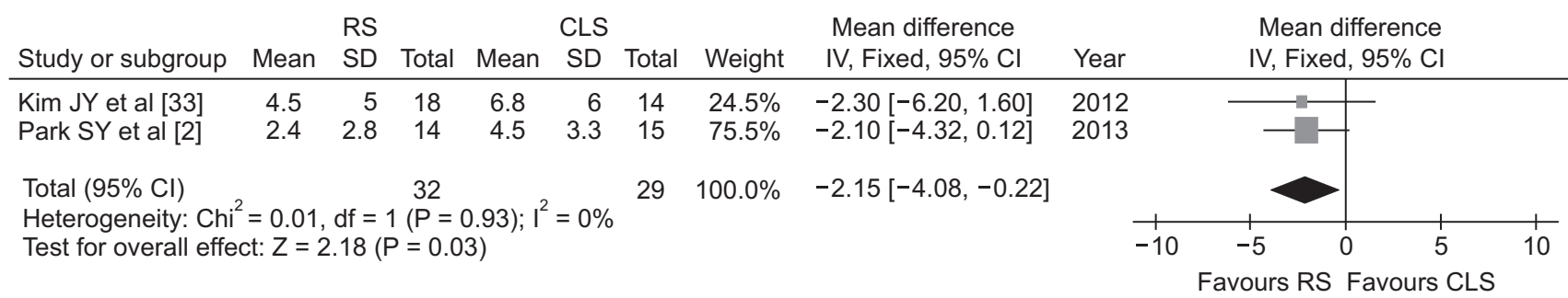

Fig. 8. Forest plot and meta-analysis of International Index of Erectile Function score change from baseline to 6 months after surgery. RS, robotic surgery; $\mathrm{CLS}$, conventional laparoscopic surgery; SD, standard deviation; $\mathrm{Cl}$, confidence interval; df, degree of freedom.

$-0.87 ; 95 \% \mathrm{CI},-1.93$ to 0.19$)$, with no heterogeneity $\left(\mathrm{P}=0.52, \mathrm{I}^{2}\right.$ $=0 \%$ (Table 2).

\section{Sexual function (IIEF)}

Sexual function was reported in two studies [2,33] comparing RS and CLS. The outcome was evaluated using IIEF with high scores related to better sexual function. In this study, only erectile function was analyzed because Park et al. [2] reported assessment of male erectile function. At three months followup, the pooled estimates using a fixed effects model showed significant difference of IIEF scores between RS and CLS (MD, $-2.82 ; 95 \% \mathrm{CI},-4.78$ to -0.87 ), with little heterogeneity ( $\mathrm{P}$ $=0.21, \mathrm{I}^{2}=37 \%$ ) (Table 2, Fig. 7). At six month three months follow-up, the pooled estimates showed a significant difference of IIEF scores between RS and CLS (MD, $-2.15 ; 95 \%$ CI, -4.08 to $-0.22)$, with no heterogeneity $\left(\mathrm{P}=0.93, \mathrm{I}^{2}=0 \%\right.$ (Table 2, Fig. 8).

\section{Publication bias}

A funnel plot analysis of the studies was performed in a meta-analysis reporting on overall postoperative complications after RS compared to those after CLS. None of the studies lay outside the $95 \% \mathrm{CI}$, and no evidence of publication bias or heterogeneity was detected among the studies except for operation time (Fig. 9).

\section{DISCUSSION}

There is currently moderate quality evidence that CLS for total mesorectal excision (TME) has similar effects as those of open TME on long-term survival outcomes for treating rectal cancer [34]. Nevertheless, CLS remains a difficult procedure to perform due to inherent limitations, including the use of straight rigid instruments within a small working area, limited freedom, the fulcrum effect, and poor ergonomics $[1,35]$. In addition, CLS has been slow to gain popularity for treating rectal cancer, as a result of the technical difficulties associated with extensively dissecting the pelvis [36]. The da Vinci Surgical System (Intuitive Surgical, Sunnyvale, CA, USA) has been popularized for performing RS because it offers technical features that help overcome such difficulties. Adopting RS has facilitated the application of minimally invasive surgery to complex surgical operations in colorectal oncology. RS for rectal cancer is a technically feasible, safe procedure and can be performed with low morbidity and a low conversion rate, even for cases with advanced rectal cancer requiring complicated, robot-assisted, lateral lymph node dissection [37].

We conducted a systematic review and meta-analysis of comparative studies published during 2008-2015 to compare safety and effectiveness between RS and CLS in patients with rectal cancer. Although meta-analyses have been published recently $[11,12,38,39]$, our study includes the most publications and reflects the latest comparative surgical results for rectal cancer. We also performed this meta-analysis to compare RS with CLS in patients with malignant diseases of the rectum only. Our results show two benefits of RS over CLS, and showed favorable urological and sexual function in RS group. 

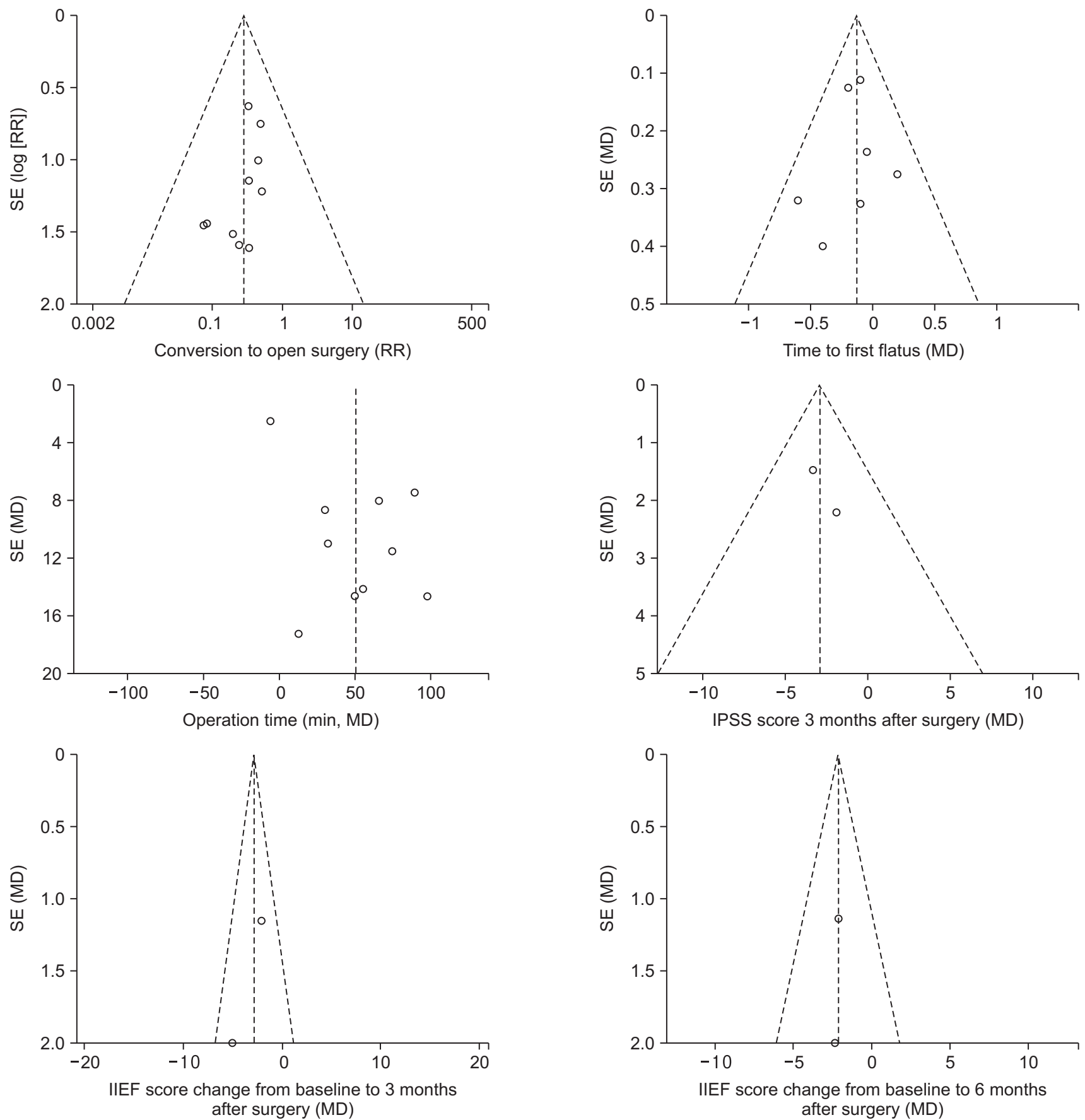

Fig. 9. Funnel plot of the studies used in the meta-analysis. IPSS, International Prostate Symptom Score; IIEF, International Index of Erectile Function; SE, standard error; $\mathrm{MD}$, mean difference; RR, risk ratio.

We found that the RS conversion rate for rectal cancer was significantly lower than that for CLS, indicating that RS provides better management of complex procedures and allowing more patients to benefit from minimally invasive surgery [39]. The reasons for conversion to open surgery included obesity with heavy mesentery, inability to identify important vascular structures, vascular injury, adhesions, and narrow pelvis; technical difficulties included stapler misfiring, inappropriate robotic arm placement, and robot malfunction [40]. The lower RS conversion rate may have been due to superior exposure and visualization of the operating field in the pelvis, thanks to the ability of the fixed fourth arm to grip and maneuver organs and the ability of the surgeon to move the three-dimensional camera as required [23]. Because conversion rate is related to a technical difficulty, surgical experience, and an intraoperative complication, it is good marker of the effectiveness of a robotic 
procedure [39]. The conversion rate for laparoscopic rectal cancer surgery is usually high, as 82 of 242 patients (34\%) with rectal cancer were converted into open surgery in the MRC CLASICC trial [1]. The most common reasons were excess tumor fixation or uncertain tumor clearance, obesity, anatomical uncertainties, and tumor inaccessibility. Conversion to open surgery increases cost [41], and the patient no longer benefits from a laparoscopic approach. Conversion results in increased blood loss, prolonged postoperative hospital stay, and higher rates of intraoperative and postoperative complications. Moreover, conversion leads to higher postoperative mortality [42] and leads to poorer survival than those of laparoscopic or open rectal surgery [43]. Therefore, the lower conversion rate is clinically significant both in the short-term and long-term.

Another major advantage of RS was the marginally significant reduction in time to first flatus. The pooled data of our study show that time to first flatus was 0.13 days shorter following RS than that after LAS. The minimally invasive characteristics and technological advantages of a robotic system may have influenced this result. Baik et al. [25] explained that the da Vinci Surgical System robotic arms are used for retraction and dissection during the TME procedure, which reduces unnecessary procedures and minimizes iatrogenic tissue injury during retraction. These differences may have been the reason for the shorter time to first flatus in RS patients, but a more advanced study is needed before such a conclusion can be drawn. However, there is little clinical significance in these small differences.

Our meta-analysis demonstrated no difference in the number of retrieved lymph nodes. TME is widely accepted as the gold standard for RS and is one of the most important factors for reducing local recurrence and to assess the oncological adequacy of resection, Further studies should compare several secondary endpoints, such as margin clearance and mesorectal fascia integrity [44].

Our study showed favorable urological and sexual function in RS group. Specifically, IPSS scores 3 months after RS were better than those of the CLS and IIEF scores change from baseline to 6 months after RS were better than CLS. Damage to hypogastric nerves or sacral splanchnic nerves affect the patient's urological and sexual functions [45,46]. Also, when the CLS is used, it is more difficult to achieve autonomic nerve preservation than RS due to insufficient tension on the dissected planes $[47,48]$.
Three-dimensional view conducted by the robotic system allowed the surgeon to better preserve the pelvic autonomic nerves. Besides, the use of the articulated mono-polar cautery hook allowed the surgeon to obtain better control of energy delivery, avoiding inopportune cauterization of the nervous bundle [19]. However, our results included a relatively small number of patients compared in both groups that the results need to be interpreted carefully.

This study had several important limitations. First, most studies in our review reported an insufficient follow-up period so many of the data were unsuitable to evaluate oncological outcomes, such as recurrence, metastasis, and mortality. Second, we could not determine whether the surgeon's learning curve affected the end result, as the studies included did not report comparisons between initial and subsequent RS experience.

In conclusion, RS provides an enhanced benefit to patients with rectal cancer in terms of postoperative recovery and the quality of the surgery. However, the quality of the evidence assembled does not support strong conclusions about most of the parameters of interest. In addition, RS is associated with a high cost and longer operating times. Therefore, findings supporting the safety and efficacy of RS need to be interpreted with caution because of the lack of RCTs. Only RCTs with a long-term follow-up could clearly determine whether the technological advantages of a robotic surgical system translate into favorable surgical and oncological outcomes. Further studies are required to evaluate the functional results and oncological outcomes associated with RS, such as the multicenter RCT of robot-assisted vs. laparoscopic resection for rectal cancer (ROLARR trial) [49].

\section{CONFLICTS OF INTEREST}

No potential conflict of interest relevant to this article was reported.

\section{ACKNOWLEDGEMENTS}

This study was funded by the National Evidence-based Healthcare Collaborating Agency in the Republic of Korea (NC13-007).

\section{REFERENCES}

1. Guillou PJ, Quirke P, Thorpe H, Walker J, Jayne DG, Smith AM, et al. Shortterm endpoints of conventional versus laparoscopic-assisted surgery in patients with colorectal cancer (MRC CLASICC trial): multicentre, randomised controlled trial. Lancet 2005:365:1718-26.

2. Park SY, Choi GS, Park JS, Kim HJ, Ryuk JP. Short-term clinical outcome of robot- 
assisted intersphincteric resection for low rectal cancer: a retrospective comparison with conventional laparoscopy. Surg Endosc 2013;27:48-55.

3. Arezzo A, Passera R, Scozzari G, Verra M, Morino M. Laparoscopy for rectal cancer reduces short-term mortality and morbidity: results of a systematic review and meta-analysis. Surg Endosc 2013;27:1485-502.

4. Sammour T, Kahokehr A, Srinivasa S, Bissett IP, Hill AG. Laparoscopic colorectal surgery is associated with a higher intraoperative complication rate than open surgery. Ann Surg 2011;253:35-43.

5. Patel VR, Thaly R, Shah K. Robotic radical prostatectomy: outcomes of 500 cases. BJU Int 2007;99:1109-12.

6. Veljovich DS, Paley PJ, Drescher CW, Everett EN, Shah C, Peters WA 3rd. Robotic surgery in gynecologic oncology: program initiation and outcomes after the first year with comparison with laparotomy for endometrial cancer staging. Am J Obstet Gynecol 2008;198:679.e1-9.

7. Weber PA, Merola S, Wasielewski A, Ballantyne GH. Telerobotic-assisted laparoscopic right and sigmoid colectomies for benign disease. Dis Colon Rectum 2002; 45:1689-94.

8. Corcione F, Esposito C, Cuccurullo D, Settembre A, Miranda N, Amato F, et al. Advantages and limits of robot-assisted laparoscopic surgery: preliminary experience. Surg Endosc 2005;19:117-9.

9. Baik SH, Kim NK, Lim DR, Hur H, Min BS, Lee KY. Oncologic outcomes and perioperative clinicopathologic results after robot-assisted tumor-specific mesorectal excision for rectal cancer. Ann Surg Oncol 2013;20:2625-32.

10. Bae SU, Saklani AP, Hur H, Min BS, Baik $\mathrm{SH}$, Lee KY, et al. Robotic and laparoscopic pelvic lymph node dissection for rectal cancer: short-term outcomes of 21 consecutive series. Ann Surg Treat Res 2014; 86:76-82.

11. Memon S, Heriot AG, Murphy DG, Bressel $\mathrm{M}$, Lynch AC. Robotic versus laparoscopic proctectomy for rectal cancer: a metaanalysis. Ann Surg Oncol 2012;19:2095-
101.

12. Trastulli S, Farinella E, Cirocchi R, Cavaliere D, Avenia N, Sciannameo F, et al. Robotic resection compared with laparoscopic rectal resection for cancer: systematic review and meta-analysis of short-term outcome. Colorectal Dis 2012;14:e134-56.

13. Xiong B, Ma L, Huang W, Zhao Q, Cheng Y, Liu J. Robotic versus laparoscopic total mesorectal excision for rectal cancer: a meta-analysis of eight studies. J Gastrointest Surg 2015;19:516-26.

14. Broholm M, Pommergaard HC, Gogenur I. Possible benefits of robot-assisted rectal cancer surgery regarding urological and sexual dysfunction: a systematic review and meta-analysis. Colorectal Dis 2015; 17:375-81.

15. Higgins JP, Green S, editors. Cochrane handbook for systematic reviews of interventions. Hoboken (NJ): WileyBlackwell; 2008.

16. Slim K, Nini E, Forestier D, Kwiatkowski F, Panis Y, Chipponi J. Methodological index for non-randomized studies (minors): development and validation of a new instrument. ANZ J Surg 2003;73:712-6.

17. Higgins JP, Thompson SG. Quantifying heterogeneity in a meta-analysis. Stat Med 2002:21:1539-58.

18. Kang J, Yoon KJ, Min BS, Hur H, Baik $\mathrm{SH}$, Kim NK, et al. The impact of robotic surgery for mid and low rectal cancer: a case-matched analysis of a 3-arm comparison: open, laparoscopic, and robotic surgery. Ann Surg 2013;257:95-101.

19. D'Annibale A, Pernazza G, Monsellato I, Pende V, Lucandri G, Mazzocchi P, et al. Total mesorectal excision: a comparison of oncological and functional outcomes between robotic and laparoscopic surgery for rectal cancer. Surg Endosc 2013; 27:1887-95.

20. Shin JY. Comparison of short-term surgical outcomes between a robotic colectomy and a laparoscopic colectomy during early experience. J Korean Soc Coloproctol 2012;28:19-26.

21. Baek JH, Pastor C, Pigazzi A. Robotic and laparoscopic total mesorectal excision for rectal cancer: a case-matched study. Surg
Endosc 2011;25:521-5.

22. Popescu I, Vasilescu C, Tomulescu V, Vasile $\mathrm{S}$, Sgarbura $\mathrm{O}$. The minimally invasive approach, laparoscopic and robotic, in rectal resection for cancer: a single center experience. Acta Chir Iugosl 2010;57:29-35.

23. Bianchi PP, Ceriani C, Locatelli A, Spinoglio G, Zampino MG, Sonzogni A, et al. Robotic versus laparoscopic total mesorectal excision for rectal cancer: a comparative analysis of oncological safety and short-term outcomes. Surg Endosc 2010; 24:2888-94.

24. Patriti A, Ceccarelli G, Bartoli A, Spaziani A, Biancafarina A, Casciola L. Short- and medium-term outcome of robot-assisted and traditional laparoscopic rectal resection. JSLS 2009:13:176-83.

25. Baik SH, Ko YT, Kang CM, Lee WJ, Kim NK, Sohn SK, et al. Robotic tumor-specific mesorectal excision of rectal cancer: short-term outcome of a pilot randomized trial. Surg Endosc 2008;22:1601-8.

26. Fernandez R, Anaya DA, Li LT, Orcutt ST, Balentine CJ, Awad SA, et al. Laparoscopic versus robotic rectal resection for rectal cancer in a veteran population. Am J Surg 2013:206:509-17.

27. Cho MS, Baek SJ, Hur H, Min BS, Baik $\mathrm{SH}$, Lee KY, et al. Short and long-term outcomes of robotic versus laparoscopic total mesorectal excision for rectal cancer: a case-matched retrospective study. Medicine (Baltimore) 2015;94:e522.

28. Baek SJ, Kim SH, Cho JS, Shin JW, Kim J. Robotic versus conventional laparoscopic surgery for rectal cancer: a cost analysis from a single institute in Korea. World J Surg 2012;36:2722-9.

29. Park JS, Choi GS, Lim KH, Jang YS, Jun SH. S052: a comparison of robot-assisted, laparoscopic, and open surgery in the treatment of rectal cancer. Surg Endosc 2011;25:240-8.

30. Patel CB, Ragupathi M, Ramos-Valadez DI, Haas EM. A three-arm (laparoscopic, hand-assisted, and robotic) matchedcase analysis of intraoperative and postoperative outcomes in minimally invasive colorectal surgery. Dis Colon Rectum 2011:54:144-50. 
31. Erguner I, Aytac E, Boler DE, Atalar B, Baca B, Karahasanoglu T, et al. What have we gained by performing robotic rectal resection? Evaluation of 64 consecutive patients who underwent laparoscopic or robotic low anterior resection for rectal adenocarcinoma. Surg Laparosc Endosc Percutan Tech 2013;23:316-9.

32. Kuo LJ, Lin YK, Chang CC, Tai CJ, Chiou JF, Chang YJ. Clinical outcomes of robotassisted intersphincteric resection for low rectal cancer: comparison with conventional laparoscopy and multifactorial analysis of the learning curve for robotic surgery. Int J Colorectal Dis 2014; 29:555-62.

33. Kim JY, Kim NK, Lee KY, Hur H, Min BS, Kim JH. A comparative study of voiding and sexual function after total mesorectal excision with autonomic nerve preservation for rectal cancer: laparoscopic versus robotic surgery. Ann Surg Oncol 2012;19:2485-93.

34. Vennix S, Pelzers L, Bouvy N, Beets GL, Pierie JP, Wiggers T, et al. Laparoscopic versus open total mesorectal excision for rectal cancer. Cochrane Database Syst Rev 2014:4:CD005200.

35. Sng KK, Hara M, Shin JW, Yoo BE, Yang KS, Kim SH. The multiphasic learning curve for robot-assisted rectal surgery. Surg Endosc 2013;27:3297-307.

36. Schwab KE, Dowson HM, Van Dellen J, Marks CG, Rockall TA. The uptake of laparoscopic colorectal surgery in Great Britain and Ireland: a questionnaire sur- vey of consultant members of the ACPGBI. Colorectal Dis 2009;11:318-22.

37. Shiomi A, Kinugasa Y, Yamaguchi T, Tomioka H, Kagawa H. Robot-assisted rectal cancer surgery: short-term outcomes for 113 consecutive patients. Int J Colorectal Dis 2014:29:1105-11.

38. Yang Y, Wang F, Zhang P, Shi C, Zou $\mathrm{Y}$, Qin $\mathrm{H}$, et al. Robot-assisted versus conventional laparoscopic surgery for colorectal disease, focusing on rectal cancer: a meta-analysis. Ann Surg Oncol 2012;19:3727-36.

39. Ortiz-Oshiro E, Sanchez-Egido I, MorenoSierra J, Perez CF, Diaz JS, FernandezRepresa JA. Robotic assistance may reduce conversion to open in rectal carcinoma laparoscopic surgery: systematic review and meta-analysis. Int J Med Robot 2012;8:360-70.

40. Alasari S, Min BS. Robotic colorectal surgery: a systematic review. ISRN Surg 2012;2012:293894.

41. Franks PJ, Bosanquet N, Thorpe H, Brown JM, Copeland J, Smith AM, et al. Shortterm costs of conventional vs laparoscopic assisted surgery in patients with colorectal cancer (MRC CLASICC trial). Br J Cancer 2006:95:6-12.

42. Yamamoto S, Fukunaga M, Miyajima N, Okuda J, Konishi F, Watanabe M, et al. Impact of conversion on surgical outcomes after laparoscopic operation for rectal carcinoma: a retrospective study of 1,073 patients. J Am Coll Surg 2009;208:383-9.

43. Rottoli M, Bona S, Rosati R, Elmore U,
Bianchi PP, Spinelli A, et al. Laparoscopic rectal resection for cancer: effects of conversion on short-term outcome and survival. Ann Surg Oncol 2009;16:1279-86.

44. Arezzo A, Passera R, Salvai A, Arolfo S, Allaix ME, Schwarzer G, et al. Laparoscopy for rectal cancer is oncologically adequate: a systematic review and meta-analysis of the literature. Surg Endosc 2015;29:334-48.

45. Hassan I, Larson DW, Cima RR, Gaw JU, Chua HK, Hahnloser D, et al. Long-term functional and quality of life outcomes after coloanal anastomosis for distal rectal cancer. Dis Colon Rectum 2006;49:1266-74.

46. Yamada K, Ogata S, Saiki Y, Fukunaga M, Tsuji Y, Takano M. Functional results of intersphincteric resection for low rectal cancer. Br J Surg 2007:94:1272-7.

47. Quah HM, Jayne DG, Eu KW, SeowChoen F. Bladder and sexual dysfunction following laparoscopically assisted and conventional open mesorectal resection for cancer. Br J Surg 2002;89:1551-6.

48. Masui H, Ike H, Yamaguchi S, Oki S, Shimada H. Male sexual function after autonomic nerve-preserving operation for rectal cancer. Dis Colon Rectum 1996; 39:1140-5.

49. Collinson FJ, Jayne DG, Pigazzi A, Tsang C, Barrie JM, Edlin R, et al. An international, multicentre, prospective, randomised, controlled, unblinded, parallel-group trial of robotic-assisted versus standard laparoscopic surgery for the curative treatment of rectal cancer. Int J Colorectal Dis 2012; 27:233-41. 